Cahiers $d u$ MONDE RUSSE

\section{Cahiers du monde russe}

Russie - Empire russe - Union soviétique et États indépendants

$51 / 4 \mid 2010$

Sciences humaines et sociales en Russie à l'Âge d'argent

\title{
Lorraine de Meaux, La Russie et la tentation de l'Orient | David Schimmelpenninck van der Oye, Russian Orientalism
}

\section{Cloé Drieu}

\section{(2) OpenEdition}

\section{Journals}

Édition électronique

URL : https://journals.openedition.org/monderusse/7429

DOI : $10.4000 /$ monderusse. 7429

ISSN : $1777-5388$

Éditeur

Éditions de l'EHESS

Édition imprimée

Date de publication : 25 novembre 2010

Pagination : 789-793

ISBN : 978-2-7132-2316-7

ISSN : $1252-6576$

Référence électronique

Cloé Drieu, «Lorraine de Meaux, La Russie et la tentation de l'Orient | David

Schimmelpenninck van der Oye, Russian Orientalism », Cahiers du monde russe [En ligne], 51/4 | 2010, mis en ligne le 09 décembre 2011, consulté le 03 septembre 2022. URL : http://

journals.openedition.org/monderusse/7429; DOI : https://doi.org/10.4000/monderusse.7429

Ce document a été généré automatiquement le 3 septembre 2022

Tous droits réservés 


\title{
Lorraine de Meaux, La Russie et la tentation de l'Orient | David Schimmelpenninck van der Oye, Russian Orientalism
}

\author{
Cloé Drieu
}

\section{RÉFÉRENCE}

Lorraine de MEAUX, La Russie et la tentation de l'Orient. Paris : Fayard, 2010, 422 p. David SCHIMMELPENNINCK VAN DER OYE, Russian Orientalism. Asia in the Russian Mind from Peter the Great to the Emigration. New Haven - Londres : Yale University Press, $2010,298 \mathrm{p}$.

Que signifie l'Orient pour la Russie ? C'est précisément à cette question, déjà posée par Dostoevskij en $1881^{1}$, que répondent ces deux ouvrages. Ceux-ci s'interrogent sur la place et la symbolique de l'Orient dans la culture, le savoir et la politique russes depuis les premières chroniques (D.Schimmelpenninck van der Oye) jusqu'à la révolution d'Octobre, avec une insistance très prononcée pour le XIX siècle, "Grand siècle russe " par excellence (L. de Meaux), où conquêtes et expansion militaire coïncident et nourrissent le dynamisme de la pensée politique, scientifique et littéraire. Vaste monde hétérogène allant du Caucase au Japon en passant par l'Asie centrale, la Mongolie, la Chine ou le monde indien, l'Orient est pour la Russie une notion géographique à la fois extérieure et intérieure. Il occupe une place essentielle dans les quêtes et questionnements identitaires, fonde la pertinence d'une mission civilisatrice russe et prouverait par là même son caractère occidental, mais il permet aussi, par un jeu de miroir, de porter un regard critique sur la conquête et l'impérialisme, et de prendre ainsi en considération la nature orientale - parce que chaotique, violente et désorganisée - de la Russie. 
2 Une démarche sensiblement similaire motive chacun des auteurs. Ils remédient d'abord, et pour la première fois, à l'une des grandes lacunes historiographiques du livre fondateur d'Edward Said qui n'abordait pas l'orientalisme russe ${ }^{2}$ (L. de Meaux, p. 55 et 94 ; D. Schimmelpenninck, p.5-6). Ensuite, prenant délibérément le contre-pied de la conception saïdienne de l'« orientalisme " en tant que discours et savoir sur l'Orient comme vecteur de domination ${ }^{3}$ (D. Schimmelpenninck préfère le terme plus neutre d'« orientology » [vostokovedenie]) - mais sans pour autant se lancer dans la polémique, ces ouvrages se donnent comme objectif de cerner la spécificité et l'ambivalence de la Russie dans son rapport à l'Orient, objet non seulement de fascination, d'inspiration et de régénérescence, mais aussi de conquête, de domination et de revanche dans la course impérialiste des puissances européennes.

Ce sont précisément les sources utilisées qui permettent de fonder cette démarche et qui nourrissent la démonstration. Dans les deux ouvrages sont abondamment convoquées les sources littéraires des grands auteurs russes (Puškin, Lermontov, Tolstoj, Dostoevskij...), les sources scientifiques des grands orientalistes et historiens russes, les sources picturales (V. Vereščagin surtout) et musicales (Borodin, Rimskij-Korsakov...). L'ouvrage de L. de Meaux fait un usage plus important des abondants mémoires et récits des militaires et officiers envoyés au Caucase (plusieurs dizaines de milliers sont recensés, ce qui permettait de pallier le manque de reconnaissance dont souffrait l'armée russe au Caucase), alors que D.Schimmelpenninck s'appuie davantage sur le renouveau historiographique russe aux États-Unis, en Russie et en France. Ils suivent tous deux un déroulé chronologique.

D. Schimmelpenninck ouvre Russian Orientalism avec un premier chapitre, intitulé « The Forest and the Steppe ", basé sur les chroniques - pour la plupart religieuses - écrites entre les $\mathrm{XII}^{\mathrm{e}}$ et le $\mathrm{xV}^{\mathrm{e}}$ siècles (Chroniques des temps passés, Dit de la campagne d'Igor, Le Voyage au-delà des trois mers, etc.) et le folklore, ce qui lui permet de déterminer les rapports hostiles ou symbiotiques entre Slaves et nomades des steppes (Petchenègues, Polovtses, Mongols et autres peuples turciques) aux époques de la Russie kiévienne puis moscovite. Sont ensuite présentés plus en détail les règnes de Pierre le Grand et de Catherine II (chapitres II et III : " The Petrine Dawn», "Catherinian Chinoiserie »), qui, pour répondre à des motivations politiques, militaires, commerciales et sous l'influence du savoir occidental, marquent le début non seulement des études orientales scientifiques mais surtout d'une vie intellectuelle et culturelle féconde inspirée par des motifs orientaux. À partir des grandes conquêtes militaires, l'intérêt pour l'Orient s'intensifie et imprègne la littérature et la peinture des créateurs, tout comme l'imaginaire des publics (ch. IV : "The Oriental Muse»). Le premier grand écrivain à découvrir et à faire découvrir le Caucase est Puškin, qui joue un rôle fondamental (suivi par Lermontov, Tolstoj et d'autres) dans la constitution d'un mythe caucasien, non sans une certaine ambiguïté parce que s'exprime aussi une critique à peine voilée de la conquête et de la "pacification ", même si l'impérialisme n'est pas radicalement remis en cause. Le savoir scientifique sur l'Orient, laïc ou missionnaire, fait ensuite l'objet des chapitres V et VI ("The Kazan School», "Missionary Orientology»): ceux-ci se structurent principalement autour de portraits d'orientalistes importants travaillant à Kazan', à l'université (Muhammad Ali Kazem-Bek) ou au sein de la Division missionnaire de l'Académie théologique (N. Il'minskij, Père Hyacinthe). Si Kazan' était, de ce fait, le centre majeur des études orientales durant la première moitié $\mathrm{du} \mathrm{XIX}^{\mathrm{e}}$ siècle, SaintPétersbourg prendra progressivement la relève, notamment avec la création en 1855 de 
la faculté des langues orientales (ch. VII et VIII : «The Rise of The Saint-Petersburg School ", "The Oriental Faculty »). Bien que les attributs de cette institution nouvelle aient été avant tout de former des cadres administratifs (formation d'officiers, diplomates, etc.), cela n'empêchait pas certains orientalistes d'envergure de se consacrer à des questions moins politiques (V. Vasil'ev pour les mondes chinois et bouddhiste, S. Ol'denburg pour l'Inde et le bouddhisme) et de faire rayonner l'orientalisme russe. Le dernier chapitre ("The Exotic Self») revient sur l'impact de l'Orient dans les arts (musique classique avec Borodin, Glinka ou la littérature avec Belyj), mais à une période plus tardive (fin du XIX siècle, et l'" Âge d'argent » au début $\mathrm{xx}^{\mathrm{e}}$ siècle).

Si l'ouvrage de D.Schimmelpenninck insiste plutôt sur les grandes figures de l'orientalisme, sur les institutions et sur la place de l'Orient dans la création, celui de L. de Meaux - tout en reprenant ces mêmes aspects - s'ancre davantage dans l'histoire des idées et l'histoire politique. Il est divisé en cinq chapitres. La Russie et la Tentation de l'Orient s'interroge sur la place et le rôle de l'Orient dans la constitution de l'identité impériale, essentiellement au xix ${ }^{\mathrm{e}}$ siècle. Dans le premier chapitre («Une épopée russe : la construction d'un Empire d'Orient au $\left.\mathrm{XIX}^{\mathrm{e}} \mathrm{s} . »\right)$, l'auteur analyse le processus de conquête au Caucase, en Asie centrale puis en Extrême-Orient, ainsi que les mythes et l'imaginaire que la domination militaire coloniale a générés. S'étirant de 1801 à 1905 (défaite dans la guerre russo-japonaise), cette phase de conquête et d'affirmation de sa puissance militaire permet à la Russie de revendiquer son " caractère occidental » et sa mission civilisatrice. Mais elle révèle aussi l'impact de l'Orient sur le vécu de ceux qui sont en première ligne - soldats, écrivains ou peintres. Le deuxième chapitre "L'invention d'une science orientaliste à l'usage de la Russie " - offre, d'une part, un tableau de la création d'institutions propres à produire un savoir sur l'Orient (Académie asiatique de S. Uvarov, École de Kazan' avec Kazem-Bek, puis transfert à SaintPétersbourg en 1854) et, d'autre part, un aperçu détaillé des explorations et études des premiers scientifiques (N. Prževal'skij, V. Radlov, etc.). Est enfin évoquée la médiation des orientalistes dans la société impériale, tantôt au service du pouvoir central, tantôt porte-parole de la précarité des sujets orientaux, ainsi que leur rôle dans la politique intérieure comme étrangère ou les milieux d'affaires. La création littéraire est au cœur du troisième chapitre : « De Pouchkine à Tolstoï : le roman de l'Orient », ce qui donne au lecteur l'opportunité de remonter aux origines des représentations russes fondatrices de l'Orient et de refléter, de façon complexe, la domination coloniale et les tensions nationales de l'Empire russe. Avec près d'un siècle de retard par rapport à l'intérêt des lettres occidentales pour l'Orient, Puškin, Lomonosov, Čehov ou Tolstoj bouleversent la littérature russe en mobilisant des motifs orientaux. Ils ouvrent la voie à un romantisme oriental qui hérite des stéréotypes de l'Orient - voluptueux, exotique, religieux ou despotique - forgés par les littératures occidentale ou persane, mais lèguent aussi des images d'Orient bien plus ambivalentes. Le regard critique de la littérature dénonce aussi le leurre colonial, l'irresponsabilité de l'aventure militaire, violente et destructrice, où l'« Orient russe » est un lieu d'exil, de souffrance. À cette analyse de la littérature succède au chapitre IV (« Des slavophiles aux eurasistes : l'Orient, obsession de l'Idée russe ») celle de la place de l'Orient dans la pensée russe, slavophile en particulier, qui s'est largement constituée contre l'Occident au XIX siècle. Le discours slavophile, qui insiste à partir de 1830 sur une altérité radicale avec l'Occident et fonde la spécificité russe sur son identité à la fois slave et chrétienne d'Orient, intègre dès les années 1880 l'expansion coloniale, justifiée par un nouveau messianisme civilisateur qui emprunte au registre religieux - ce qui sera récupéré, sous une autre forme, par les bolcheviks. La 
revendication quant à l'asiatisme des Russes ainsi que les théories élaborées pour affirmer la proximité de la Russie et de l'Asie permettent, selon les auteurs, de considérer les Russes comme les héritiers des grandes civilisations (aryenne, scythe, grécohellénique, turco-mongole). Ces considérations tentent de justifier l'impérialisme et s'accompagnent de sentiments hostiles de plus en plus prononcés à l'égard de l'islam et des musulmans de l'Empire ottoman ou de Russie, malgré la formulation de l'idéal d'un empire musulman-chrétien professé par les Tatars réformateurs, Ismaïl bey Gaspyraly (Gasprinskij) en particulier. Ces tensions grandissantes et les invocations de plus en plus contradictoires de l'Orient dans la réflexion sur l'identité impériale russe rendent largement comptent de la "confusion" de cette dernière à la veille de la Première Guerre mondiale. Analysés au chapitre V, intitulé «Le 'style russe': un art métissé en héritage ", les métissages culturels dans la musique de style russo-oriental ou la peinture font rayonner la culture russe jusqu'en Occident. Mais, au tournant du $\mathrm{xx}^{\mathrm{e}}$ siècle, ces métissages présagent peut-être aussi, à leur façon, des bouleversements à venir. Qu'elle soit considérée comme venant d'Occident ou d'Orient, la menace ou la promesse révolutionnaire semble imminente.

6 Comme le démontrent ces deux ouvrages, l'Orient est pour la Russie une ressource identitaire, culturelle, politique ou scientifique. Il est convoqué de façon variable en fonction d'événements fondateurs, constitués le plus souvent par des conquêtes ou défaites militaires : la maitrise des territoires centrasiatiques représente ainsi une étape décisive de l'« occidentalisation» russe, alors que les défaites dans la guerre de Crimée (1853-1856) ou dans la guerre russo-japonaise rappellent son arriération technique et militaire et, de fait, son caractère «oriental» pour l'Europe. Le "projecteur orientaliste" (de Meaux, p.242) rend donc compte avec précision des profondes mutations de la vie politique, culturelle et scientifique que l'Empire russe traverse jusqu'à la révolution d'Octobre. Si la lecture des ouvrages est particulièrement agréable, stimulante et les démonstrations convaincantes, on pourrait cependant objecter qu'en choisissant délibérément la focale anti-saïdienne et en insistant sur le dualisme intrinsèque de l'identité russe, les auteurs font l'économie d'une analyse plus approfondie des rapports entre "Orient», "discours politique» et "pouvoir». La bivalence de l'identité russe ne fonderait-elle pas aussi un "rapport de domination et d'autorité» sur des régions conquises? Comment cette bivalence est-elle instrumentalisée ? Ainsi, il aurait été intéressant de cerner davantage les décalages entre « discours sur l'Orient » et « discours politique » d'une part, « orientalisme » et « culture populaire » d'autre part. Si les images de l'Orient composées par les écrivains, les peintres ou les savants - la « haute culture » - reflètent les contradictions de leur temps, qu'en est-il des représentations de l'Orient véhiculées dans les nouvelles, les feuilletons et toute autre production culturelle populaire?

7 Quoi qu'il en soit, ces deux ouvrages permettent de saisir toute la complexité, la diversité et la singularité du rapport de la Russie à l'Orient sur le temps long. Il faut d'ailleurs saluer le fait que, tout en restant principalement focalisés sur la période impériale, ils proposent quelques «lignes de fuite» à partir de la période révolutionnaire et les années qui suivent, constituant indéniablement une base solide pour élargir le champ chronologique à l'ensemble de la période soviétique afin de déterminer les ruptures et continuités des représentations de l'Orient et de son instrumentalisation dans le discours politique et les productions culturelles. L'invocation d'Edward Said ${ }^{4}$ - que les ouvrages s'en inspirent ou s'en démarquent permet d'interroger toujours plus la nature de la domination russe (impériale ou 
soviétique) et sa marginalité relative en replaçant cette réflexion dans un contexte européen et comparatiste.

\section{NOTES}

1. Il s'agit du texte «Geok-Tepe, čto takoe dlja nas Azija ?» [Geok-tepe : que signifie pour nous l'Asie ?], publié dans Dnevnik pisatelja [Journal d'un écrivain].

2. E. Said reconnaît explicitement ne pas rendre justice à l'importante contribution de l'Allemagne, de l'Italie, du Portugal, de l'Espagne et de la Russie, Edward Said, Orientalisme, L'Orient créé par l'Occident, P. : Seuil, 2003 [1978, 1995], p. 30.

3. L'acception saidienne étroite d'« orientalisme » se définit comme " savoir sur l'Orient». Dans son acception plus large, ce terme se définit comme "style de pensée fondé sur la distinction ontologique et épistémologique entre "Orient" et (le plus souvent) "Occident" » et comme "style occidental de domination, de restructuration et d'autorité sur l'Orient», (Said, Orientalisme, p. 14-15). C'est quasiment en ces termes, une vingtaine d'années plus tôt, que la Grande Encyclopédie soviétique avait défini l'«orientalisme occidental et bourgeois » qui "subordonn[ait] l'étude de l'Orient aux politiques coloniales des puissances impérialistes ", «Orientalisme » [Vostokovedenie] (notice), Bol'šaja Sovetskaja Enciklopedija, 1951 (2 éd.), cité par D. Schimmelpenninck van der Oye, Russian Orientalism, p. 6.

4. L'ouvrage a été traduit en russe seulement en 2006: voir à ce sujet l'article de Vladimir Bobrovnikov, «Počemu my marginaly? Zametki na poliah russkogo perevoda 'Orientalisma' Edvarda Saida » [Pourquoi sommes-nous marginaux ? Remarques sur la traduction en russe d'Orientalisme d'Edward Said], Ab Imperio, 2 (2008), p. 325-344. 